\title{
Hot-electron transport and magnetic anisotropy in epitaxial spin valves
}

\author{
E. Heindl, J. Vancea, G. Woltersdorf, and C. H. Back \\ Department of Physics, Universität Regensburg, 93040 Regensburg, Germany
}

(Received 6 June 2007; published 27 September 2007)

\begin{abstract}
We report on ballistic electron magnetic microscopy studies at room temperature using an epitaxially grown $\mathrm{Fe}_{34} \mathrm{Co}_{66} / \mathrm{Au} / \mathrm{Fe}_{34} \mathrm{Co}_{66}$ trilayer. Local hysteresis loops are obtained as a function of the in-plane magnetic field angle. In order to understand the underlying local magnetization behavior, the magnetic anisotropies were determined by ferromagnetic resonance. These results served as input for simulations of the hysteresis loops, which are compared to magneto-optic Kerr effect and ballistic electron magnetic microscopy data of the spin valve. In doing so, the relative magnetization configuration of the spin valve can be calculated as a function of the external magnetic field, and the magnetization behavior during the reversal can be explained. Since different magnetization configurations of the spin valve are available, epitaxial spin valves allow multimagnetocurrent values, when the magnetic field is applied along different directions.
\end{abstract}

DOI: $10.1103 /$ PhysRevB.76.104435

PACS number(s): 75.47. $-\mathrm{m}, 76.50 .+\mathrm{g}$

The widespread interest of introducing the spin as a new degree of freedom in next generation electronic devices has strongly stimulated the investigation of magnetoresistive (MR) effects. However, only the giant magnetoresistance $(\mathrm{GMR})^{1,2}$ and the tunnel magnetoresistance $(\mathrm{TMR})^{3}$ effects seem to be reasonably applicable in magnetic recording devices so far. Other proposed devices are not competitive with existing semiconductor devices yet, since the generation, manipulation, and detection of the spin polarization is more challenging than the usage of the charge only. A commonly used spintronic device for these tasks is the spin valve in which the spin transport is directly related to the magnetization configuration. The spin valve is involved in GMR and TMR elements and also in hot-electron devices, such as spin valve transistors (SVT's) ${ }^{4}$ and magnetic tunnel transistors (MTT's). ${ }^{5}$ The latter two devices are based on ballistic electron transport. Thus, mostly electrons, which do not suffer any scattering during the transport through the spin valve, create the output current of such devices. The largest spin filter or spin valve effect is obtained when single domain states combined with a collinear magnetization alignment of the two ferromagnetic layers are involved. Multidomain states or deviations from collinear magnetization alignment often occur in the vicinity of the coercive fields of the individual magnetic layer and can drastically reduce the MR effect. In order to optimize hot-electron devices, the magnetization configuration and spin transport characteristics should be determined with a high lateral resolution. We employ the ballistic electron magnetic microscopy (BEMM), which is a powerful method due to its nanometer resolution and the variable hot-electron energy. ${ }^{6-12}$

This technique is related to the ballistic electron emission microscopy (BEEM), invented by Bell and Kaiser in 1988 as an extension of scanning tunneling microscopy. ${ }^{13}$ In the case of magnetic samples, BEEM is often referred to as BEMM. ${ }^{7}$ During operation, a scanning tunneling microscope (STM) tip (emitter) injects nonequilibrium charge carriers into the spin valve (base) grown onto a $n$-type semiconductor (collector). In this way, the study of the spin dependent hotelectron transport as well as the imaging of magnetic domains in spin valves by recording the collector current in an external magnetic field are possible. ${ }^{6-9}$ In our convention, the sign of the collector signal is positive for electrons flowing from the base into the semiconductor. The injected hot electrons undergo a variety of elastic and inelastic scattering processes in the metallic layers. Ferromagnetic layers exhibit spin dependent electron scattering and spin dependent electron group velocities. ${ }^{14,15}$ This spin dependence manifests itself by spin dependent ballistic mean free paths or hotelectron attenuation lengths and in turn leads to a strongly spin polarized current entering the semiconductor collector. The attenuation length $\lambda$, defined as the layer thickness decreasing the collector current by a factor of $e^{-1}$, is much larger for majority spin hot electrons than for minority spins. ${ }^{8,16}$ Hence, in spin valves, the collector current is drastically reduced in antiparallel magnetization alignment compared to parallel alignment. The change of collector current between these two states is defined as the magnetocurrent MC $=\left(I_{\uparrow \uparrow}-I_{\uparrow \downarrow}\right) / I_{\uparrow \downarrow}$, with $I$ denoting the transmitted current and the indices $\uparrow \uparrow$ and $\uparrow \downarrow$ denoting parallel and antiparallel magnetization configurations, respectively. The Schottky barrier at the base-collector interface acts as an energy and momentum filter for the injected electrons. Only electrons with sufficient energy to overcome the barrier and with a suitable $\mathbf{k}$ vector, determined mainly by the band structure of the semiconductor, can enter the collector and contribute to the collector current. These electrons propagate almost perpendicular to the base-collector interface within a nanometer wide cone, which represents the lateral probe and gives rise to the high spatial resolution. Electrons which do not fulfill these two conditions are reflected at the metal-semiconductor interface.

Previous studies of polycrystalline spin valves addressed by BEMM, SVT, and MTT studies ${ }^{11,12,14,16-20}$ did not investigate the hysteresis loop as a function of the in-plane applied magnetic field angle. This is due to the fact that a polycrystalline structure often leads to a cancellation of the magnetic anisotropy. Therefore, in-plane hysteresis loops are expected to be mostly independent of the angle of the applied magnetic field. On the other hand, epitaxial or textured ferromagnetic layers may exhibit an in-plane magnetocrystalline anisotropy and allow the detailed investigation of the switching processes. 
In this work, we present BEMM studies of an epitaxial $\mathrm{Fe}_{34} \mathrm{Co}_{66} / \mathrm{Au} / \mathrm{Fe}_{34} \mathrm{Co}_{66}$ spin valve and its magnetic switching behavior as a function of the angle of the in-plane applied magnetic field. To understand the underlying local magnetization behavior, we extracted the magnetic anisotropies from ferromagnetic resonance (FMR) measurements. In addition, the macroscopic magnetic behavior was determined by angle dependent magneto-optic Kerr effect (MOKE) studies. Complementary, the magnetization orientation at different external magnetic fields is calculated by hysteresis loop simulations, which are based on the anisotropy constants obtained from FMR. The used techniques (MOKE and BEMM) depict the magnetization behavior on macroscopic and microscopic length scales. The magnetic switching fields of the spin valve determined by these two techniques agree with each other within our experimental accuracy. Nevertheless, local variations of the switching fields of order of about $40 \%$ were observed in BEMM. All experiments presented here were performed at ambient conditions. Since BEMM is a local technique with nanometer resolution and magnetic domains usually span larger length scales, we performed local hysteresis loops on several sample locations on millimeter scales.

The $\mathrm{GaAs}_{67} \mathrm{P}_{33}$ substrate consists of a $7 \mu \mathrm{m}$ thick $\mathrm{GaAs}_{67} \mathrm{P}_{33}(100)$ layer with a doping level of $n=5$ $\times 10^{16} \mathrm{~cm}^{-3}$ epitaxially grown onto a $n$-doped $\operatorname{GaAs}(100)$ wafer with $n=10^{18} \mathrm{~cm}^{-3}$. Prior to the metal deposition, the semiconductor surface has been cleaned by sputtering with $1 \mathrm{keV}$ Ar ions while simultaneously annealing at $870 \mathrm{~K}$ in UHV conditions. This process has been continued until diffraction spots using high energy electrons (reflection highenergy electron diffraction) form Bragg peaks on a circle representing large atomically flat terraces on the surface. The layer stack of $5 \mathrm{~nm} \mathrm{Au} / 2.9 \mathrm{~nm} \mathrm{Fe}{ }_{34} \mathrm{Co}_{66} / 3.6 \mathrm{~nm} \mathrm{Au} / 2.9 \mathrm{~nm}$ $\mathrm{Fe}_{34} \mathrm{Co}_{66}$ has been successively evaporated by molecular beam epitaxy at room temperature. Evidence for the fully epitaxial growth is reported elsewhere. ${ }^{10}$ In order to keep the leakage currents flowing into the semiconductor small, the sample was cut so that the effective diode area is about 1 $\times 2 \mathrm{~mm}^{2}$. Throughout the paper, we label the $\mathrm{Fe}_{34} \mathrm{Co}_{66}$ layer deposited directly onto the semiconductor substrate as FM1 and the second $\mathrm{Fe}_{34} \mathrm{Co}_{66}$ layer as FM2.

Layers of $\mathrm{Fe}_{x} \mathrm{Co}_{1-x}$ with $x>0.2$ form a stable body centered cubic lattice ${ }^{21}$ and as previously reported for single crystalline $\mathrm{Fe}_{34} \mathrm{Co}_{66}$ layers on $n$-GaAs(100), the [110] direction represents the magnetic easy axis. ${ }^{22,23}$ This orientation is due to the angular dependence of the fourfold magnetocrystalline anisotropy of a body centered lattice and the uniaxial contribution from the $\mathrm{Fe}_{34} \mathrm{Co}_{66} / \mathrm{GaAs}(100)$ interface. ${ }^{22,23}$ For a $\mathrm{Fe}_{34} \mathrm{Co}_{66}$ layer grown onto the related III-V semiconductor $\mathrm{GaAs}_{67} \mathrm{P}_{33}(100)$, one can expect similar magnetic anisotropies with the same easy axis along [110]. For the FM2 layer, however, only the fourfold crystal anisotropy should be present and two easy axes along [110] and [1-10] can be expected.

To gain insight into the magnetic behavior of the spin valve during the magnetization reversal, the magnetic anisotropies were determined by FMR measurements. The rf magnetic field was applied in the film plane but perpendicu- a)
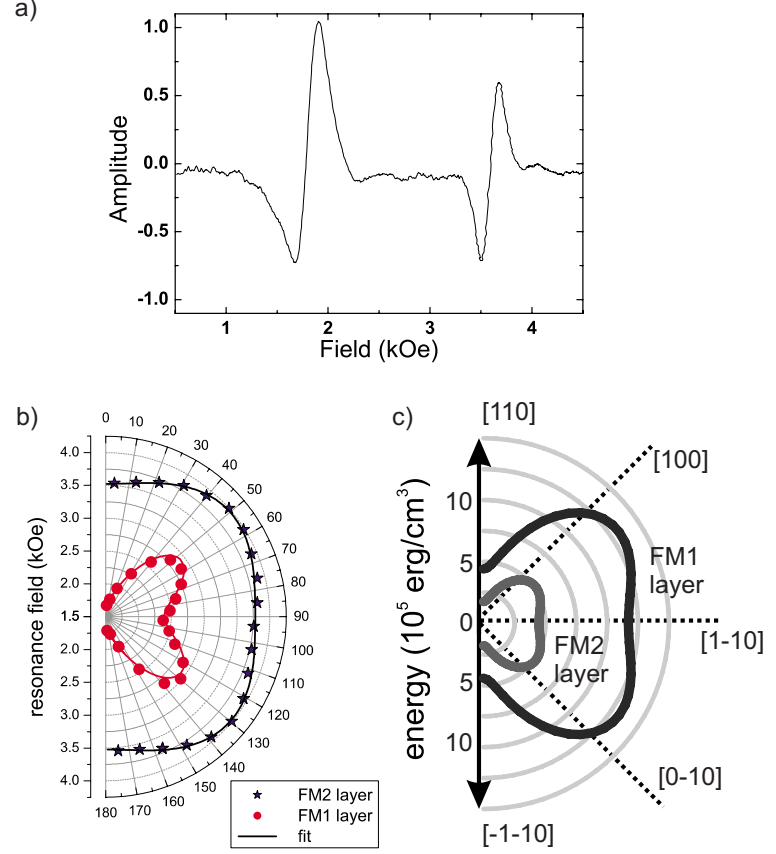

FIG. 1. (Color online) (a) Typical ferromagnetic resonance measurement of the spin valve showing two distinct resonances, which show up as derivatives of Lorentz curves. (b) Angular dependence of the FMR fields of the spin valve with respect to the [110] axis and fit with the linearized Landau-Lifshitz equation of motion (solid line). (c) Energy landscape for the magnetization: contributions of a uniaxial and a fourfold crystal anisotropy component are present in the two $\mathrm{Fe}_{34} \mathrm{Co}_{66}$ layers.

lar to the dc magnetic field. By measuring the FMR fields as a function of the in-plane applied field angle $\Phi_{H}$ with respect to the [110] orientation, the magnetic anisotropies were determined. In Figs. 1(a) and 1(b), the low field resonance corresponds to the layer, with the larger magnetic anisotropy, i.e., the FM1 layer and the high field resonance to the FM2 layer, respectively. The FMR data were analyzed using the linearized Landau-Lifshitz equation of motion ${ }^{24,25}$ and the effective anisotropy constants $K_{1}^{\mathrm{eff}}$ and $K_{u}^{\mathrm{eff}}$ are obtained:

\begin{tabular}{lcc} 
& FM1 layer & FM2 layer \\
\hline$K_{u}^{\text {eff }}\left(\mathrm{erg} / \mathrm{cm}^{-3}\right)$ & $3.7 \times 10^{5}$ & $1.4 \times 10^{5}$ \\
$K_{1}^{\text {eff }}\left(\mathrm{erg} / \mathrm{cm}^{-3}\right)$ & $4.4 \times 10^{5}$ & $1.8 \times 10^{5}$
\end{tabular}

Note that the anisotropy constants contain bulk and interface contributions. Figure 1(c) shows the energy landscape for the magnetization calculated with the obtained anisotropy constants according to Ref. 24. Both $\mathrm{Fe}_{34} \mathrm{Co}_{66}$ layers exhibit an energy landscape of even shape composed of a four- and a twofold component. The magnetic easy axis for both $\mathrm{Fe}_{34} \mathrm{Co}_{66}$ layers is given by the global minima in Fig. 1(c) along the [110] axis. The fourfold component can be attributed to the crystal anisotropy and the twofold component in the FM1 layer to the uniaxial anisotropy of the $\mathrm{Fe}_{34} \mathrm{Co}_{66^{-}}$ $\mathrm{GaAs}_{67} \mathrm{P}_{33}$ interface. An additional uniaxial anisotropy component with easy axis along [110] is also recognizable for the FM2 layer. This can be caused by lattice strain or additional 
a)

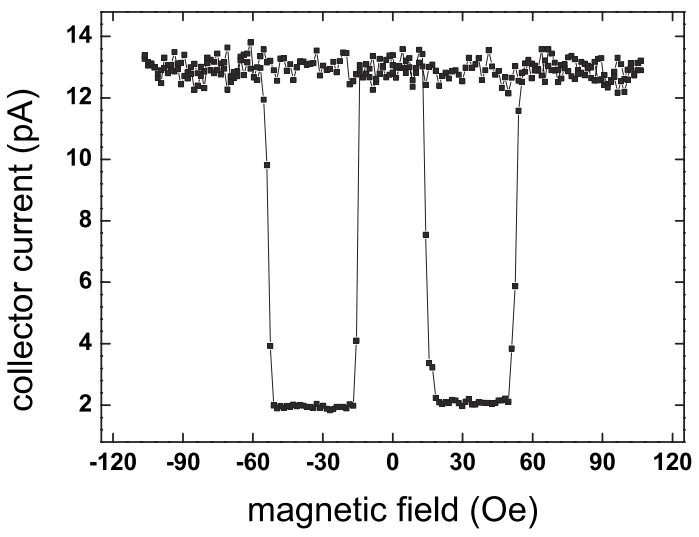

b)

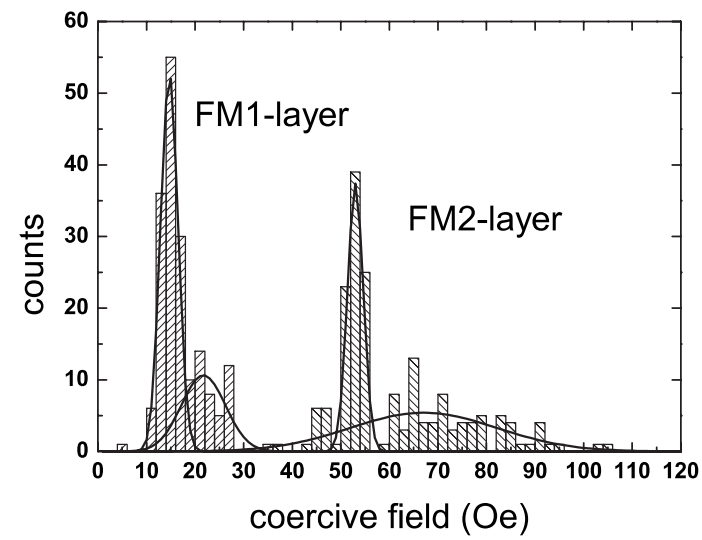

FIG. 2. Field sweep along the easy [110] axis: (a) Local hysteresis loop obtained by BEMM at tunneling voltages of $-2 \mathrm{~V}$ and at tunneling currents of $20 \mathrm{nA}$. (b) Coercive field distribution measured at several locations on the sample by BEMM.

interface anisotropies. ${ }^{26,27}$ Since the global minima of the crystal anisotropy as well as of the uniaxial interface anisotropy are along the [110] axis, the magnetic easy axis is well defined along [110], as can be seen in the orientation of the energy landscape shown in Fig. 1(c).

In Fig. 2(a), we present a typical local hysteresis loop obtained by BEMM. The external magnetic field has been swept along the magnetic easy [110] axis. The experimental parameters were $-2 \mathrm{~V}$ for the tunneling voltage and $20 \mathrm{nA}$ for the tunneling current. The local hysteresis loop of Fig. 2(a) exhibits two plateaus, which represent the parallel and the antiparallel magnetization configuration, respectively. We were also able to obtain local hysteresis loops of similar shape on other sample locations but with switching fields partially deviating by more than $40 \%$, as can be seen in Fig. 2(b). The sample locations were randomly selected from the whole sample area. A change of the tunneling location on the sample was performed by the translation of the sample with a three-axis piezo positioner ${ }^{28}$ such that a constant relative angle between sample and magnetic field is maintained. The statistical distribution of the coercive fields can be fitted by a combination of two Gaussian curves for each ferromagnetic layer. The mean coercive field values with the corresponding standard deviations were determined to be

$$
H_{c}(\mathrm{FM} 1)=\left\{\begin{array}{c}
15 \pm 40 \mathrm{Oe} \\
22 \pm 9 \mathrm{Oe}
\end{array}\right.
$$

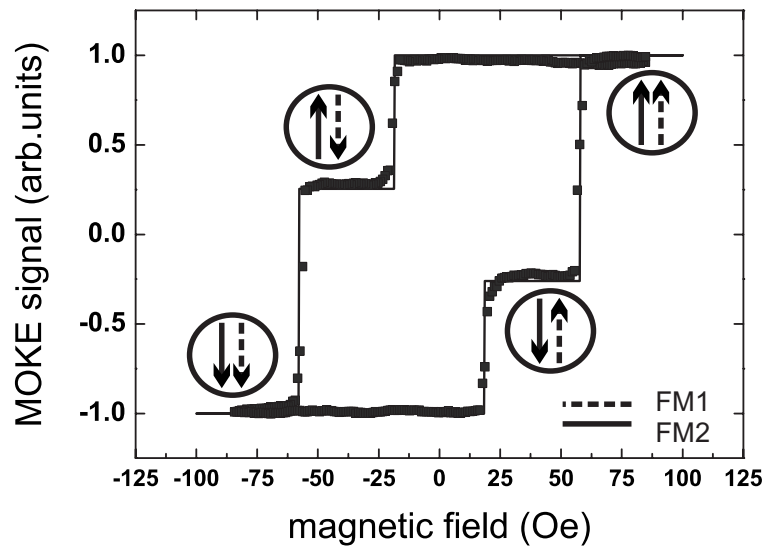

FIG. 3. Field sweep along the easy [110] axis: Magneto-optic Kerr effect measurement and simulation (solid line) with calculated magnetization configurations.

$$
H_{c}(\mathrm{FM} 2)=\left\{\begin{array}{c}
53 \pm 30 \mathrm{Oe} \\
70 \pm 30 \mathrm{Oe} .
\end{array}\right.
$$

The mean values of the low field Gaussians represent the most frequently occurring coercive fields and match well the switching fields of the local hysteresis loop of Fig. 2(a) $\left(H_{c}^{\mathrm{FM} 1}=15 \mathrm{Oe}\right.$ and $\left.H_{c}^{\mathrm{FM} 2}=53 \mathrm{Oe}\right)$. The appearance of two Gaussian distributions is an evidence for the existence of two different kinds of magnetic domains. The low coercive field peak is related to weakly pinned domain walls and the larger coercive fields correspond to more strongly pinned ones. Since this behavior is found in both layers, the origin of the pinning can be attributed to defects ranging through both ferromagnetic layers, e.g., misfit dislocations. The depinning from these defect sites is different for both layers due to the different magnetic anisotropies. We performed numerous scans within our largest scanning range of about $4 \mu \mathrm{m}^{2}$ with the aim to image magnetic domains using BEMM. Before starting the scan process, the external magnetic field was adjusted to field values of 14 and 49 Oe, respectively. After each scan, the field was increased by field steps of 0.7 Oe. However, we often observed domain wall motion underneath the STM tip during the scan process. This observation indicates and confirms to some extent the existence of at least micrometer-scale domains, which are easily movable, i.e., weakly pinned.

The two highest Gaussian peaks of Fig. 2(b) are also reflected in MOKE measurements (see Fig. 3). MOKE probes a macroscopic sample area compared to BEMM and thus averages the coercive fields over this area. The corresponding hysteresis curve exhibits switching fields of $H_{c}^{\mathrm{FM} 1}$ $=19 \mathrm{Oe}$ and $H_{c}^{\mathrm{FM} 2}=57$ Oe which match well the data from Figs. 2(a) and 2(b). The rectangular shape and the sharp two step switching behavior of the MOKE curve indicate a well defined parallel and antiparallel magnetization configuration of the spin valve and a magnetization reversal dominated by domain wall motion processes. The fingerprint of the domain wall motion is the sharp change in the local hysteresis loop or the sharp change in the MOKE data. Since both $\mathrm{Fe}_{34} \mathrm{Co}_{66}$ layers have the same thickness, the smaller Kerr rotation, i.e., 
a)

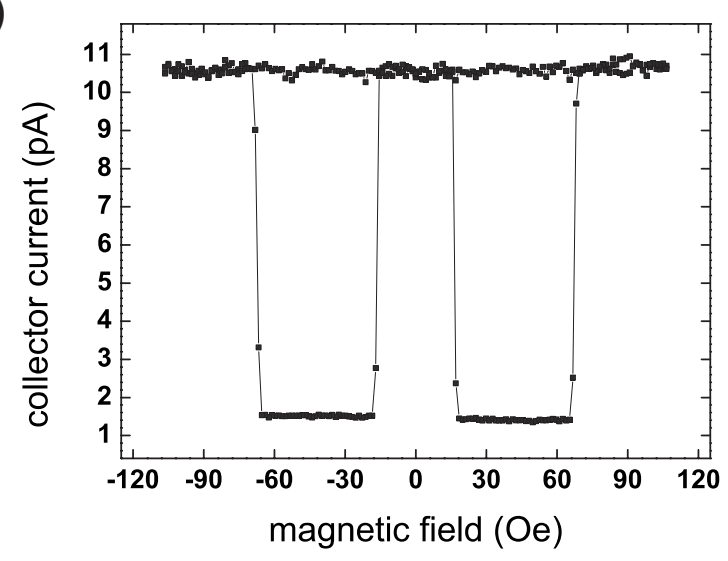

b)

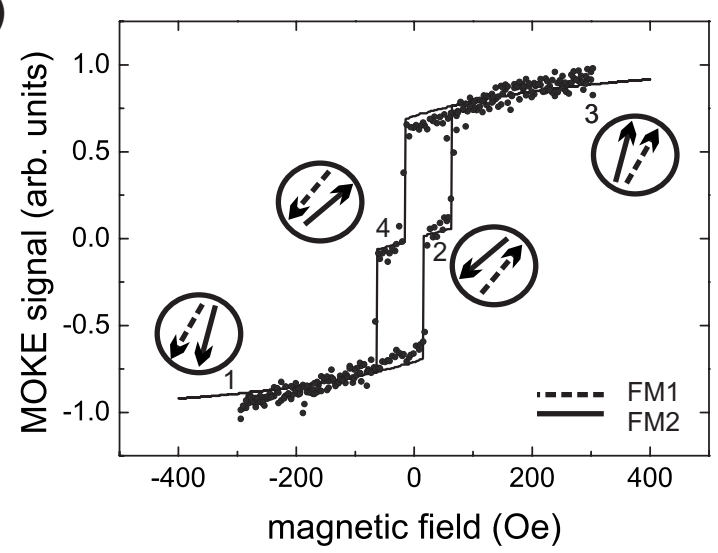

FIG. 4. Field sweep along the hard [100] axis: (a) Local hysteresis loop obtained by BEMM at tunneling voltages of $-1.7 \mathrm{~V}$ and at tunneling currents of $20 \mathrm{nA}$. (b) Magneto-optic Kerr effect measurement and simulation (solid line) with calculated magnetization configurations.

the lower magnetic switching field, is attributed to the FM1 layer. In order to achieve a quantitative statement about the quality of the magnetization alignments, we simulate the hysteresis loops with the already determined magnetic anisotropies and calculate the most interesting magnetization configurations of the spin valve (Fig. 3, solid line). In the simulations, the domain wall energies are the only free parameters. They are adjusted such that the simulated curve shows the same coercive fields as in the experimental results. With the field sweep along this easy [110] axis only, almost perfectly collinear magnetization configurations of the spin valve are reached, which was already indicated by the rectangular shape of the local hysteresis loop from BEEM [Fig. 2(a)] and the macroscopic MOKE loop in Fig. 3.

In a second set of BEMM experiments, the external magnetic field was applied and swept along the [100] axis. With respect to the FMR results of Fig. 1, this direction represents the magnetic hard axis for both ferromagnetic layers. In Fig. 4(a), a typical local hysteresis loop obtained by BEMM is shown. The experimental parameters were $-1.7 \mathrm{~V}$ for the tunneling voltage and $20 \mathrm{nA}$ for the tunneling current. The magnetocurrent reaches almost the same value of $600 \%$ as in the loop obtained during reversal along the easy axis, ${ }^{10}$ showing that the relative orientation of the local magnetiza- tions is almost parallel or antiparallel during the reversal. Figure 4(b) shows the corresponding MOKE curve. With the already determined magnetic anisotropies, this hysteresis curve was also simulated and adjusted to the experimental data [see Fig. 4(b), solid line]. Starting from saturation and decreasing the magnetic field, one clearly recognizes a rotation of the magnetizations of the two layers out of the [100] direction toward the neighboring minimum in the energy landscape, e.g., to the magnetic easy [110] axis. With sufficiently large opposing field, the FM1 layer switches by an angle of about $180^{\circ}$ via domain wall motion processes. A further increase of the field leads to magnetization rotation processes toward the $[-100]$ direction. The same behavior can be observed for the FM2 layer but with coercive fields shifted to larger absolute values. The corresponding coercive fields are 18 and 67 Oe for the FM1 and FM2 layers, respectively. The fact that the influence of the magnetization rotation is not observed in the local hysteresis loop from BEMM is a result of a nearly parallel rotation process. BEMM is only sensitive to the relative angle between the two magnetizations. In addition, the collector current corresponds only to the cosine of this angle, which further reduces the sensitivity at small deviations from antiparallel or parallel magnetization configuration. The switching fields of $H_{c}^{\mathrm{FM} 1}=17 \mathrm{Oe}$ and $H_{c}^{\mathrm{FM} 2}=67 \mathrm{Oe}$, determined from Fig. 4(a), are again consistent with the MOKE results but are significantly larger compared to the magnetization reversal along the easy axis.

Finally, magnetic field sweeps along the [1-10] axis give rise to the local hysteresis loop shown in Fig. 5(a). From the FMR measurements, it is clear that this direction represents an intermediate magnetic axis for both ferromagnetic layers. The experimental parameters were $-2 \mathrm{~V}$ for the tunneling voltage and $20 \mathrm{nA}$ for the tunneling current. At first glance, three plateaus are visible, which can be attributed to a parallel, an antiparallel, and a $90^{\circ}$ configuration of the spin valve. The corresponding MOKE curve is shown in Fig. 5(b). For clarity, only one sweep direction is shown. In contrast to the local hysteresis loop, no sharp magnetic switching and plateaus are visible in the macroscopic MOKE curve. We can, however, observe four humps each with a steep and softened step and, additionally, a nonvanishing slope indicating magnetization rotation processes at all external fields. The two humps around remanence belong to the FM2 layer. In the BEMM setup, only fields up to 150 Oe can be generated. Thus, the local hysteresis loop in Fig. 5(a) represents a reversal without reaching the saturation state. We now proceed to simulate the hysteresis curve [points 1-6 in Fig. 5(b)] and extract the magnetization configuration at the most interesting external field values. With an almost $90^{\circ}$ configuration of the spin valve at fields of \pm 150 Oe and a parallel (points 2 and 3) and an antiparallel (points 4 and 5) configuration at field values around remanence, the simulated loop reproduces satisfactorily the local hysteresis loop at most points during the magnetization reversal [compare Fig. 5(c)]. However, at an external magnetic field of about \pm 70 Oe, Fig. 5(a) shows a sharp change of the magnetization configuration and differs therefore significantly from the simulation in Fig. 5(c). This difference can be well explained in terms of small angle deviations from the [1-10] orientation of the external field in the BEMM experiment. In Figs. 6(a) and 6(b), the 
a)

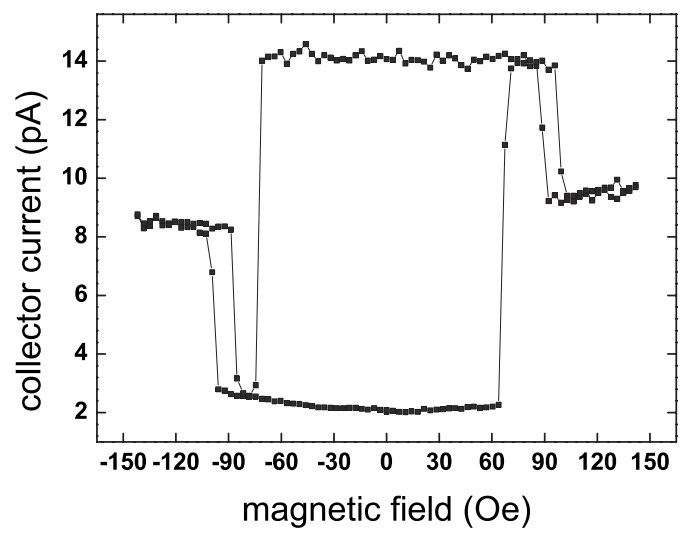

b)

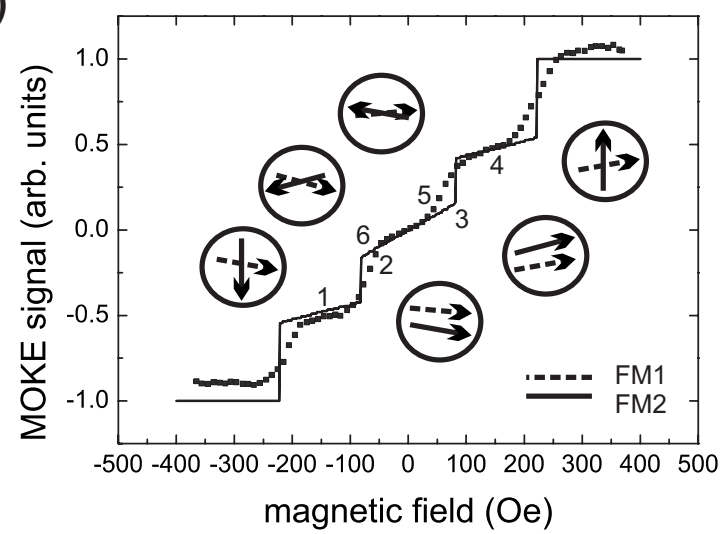

c)

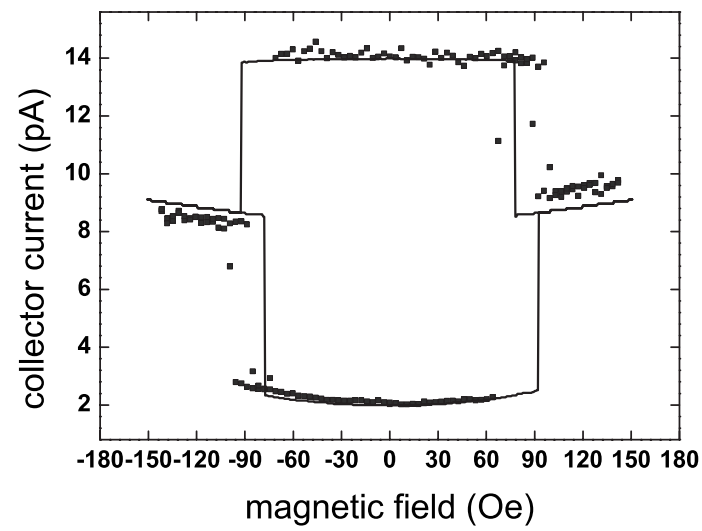

FIG. 5. Field sweep along the intermediate [1-10] axis: (a) Local hysteresis loop obtained by BEMM at tunneling voltages of $-2 \mathrm{~V}$ and at tunneling currents of $20 \mathrm{nA}$. (b) Magneto-optic Kerr effect measurement and simulation (solid line) with calculated magnetization configurations. Simulation of the local BEMM hysteresis loop.

resulting MOKE and BEMM loops accompanied by the calculated magnetization configurations [points 1-6 in Fig. $6(\mathrm{a})]$ are shown when the external field is swept along an angle of $2^{\circ}$ with respect to the [1-10] axis. These simulations explain the BEMM loop of Fig. 5(a). At 150 Oe, the magnetization of the FM2 layer is orientated parallel to the magnetic field, e.g., parallel to the [1-10] axis, while the magnetization from the FM1 layer is orientated near the [110] axis, such that a magnetization configuration with a relative angle of approximately $90^{\circ}$ is reached. Reducing the external field
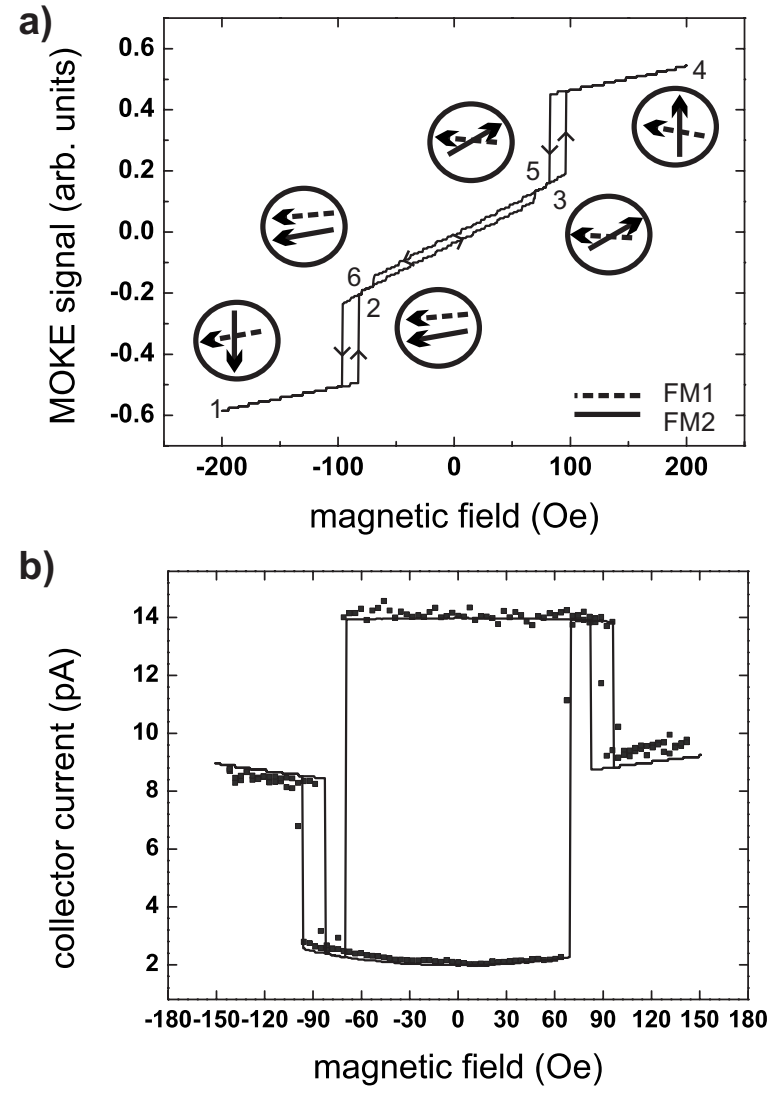

FIG. 6. Field sweep along a direction deviating by $2^{\circ}$ from the [1-10] axis: (a) MOKE hysteresis loop simulation (solid line) with calculated magnetization configurations. (b) Local hysteresis loop obtained by BEMM at tunneling voltages of $-2 \mathrm{~V}$ and at tunneling currents of $20 \mathrm{nA}$ and simulation (solid line).

leads to a coherent rotation of the spins in both ferromagnetic layers but predominantly in the FM1 layer, which is indicated by the slope in the experimental local hysteresis loop of Fig. 5(a) and in the macroscopic MOKE loop of Fig. 5(b). While at an external field of 150 Oe the FM2 layer magnetization is trapped between energy maxima in the $\langle 100\rangle$ directions arising from the fourfold crystal anisotropy, the FM1 layer magnetization is orientated between the [110] and, e.g., the [100] axis. Reducing the external field forces the FM1 layer magnetization to relax toward the [110] axis. Further reduction of the external field allows the FM2 layer magnetization to overcome the above mentioned barriers by relaxation in magnetic domains rather than coherent rotation processes, evidenced by the sharp change in the BEMM hysteresis loop at an external field of 89 Oe or the sharp change in Figs. 6(a) (point 2) and 6(b). In BEMM, the domain magnetization, probed by the STM tip, was orientated along the [110] axis, resulting in a parallel magnetization configuration at fields below 89 Oe. Sweeping the field from 89 to $-75 \mathrm{Oe}$, the magnetization configuration remains apparently almost parallel, since no change in the collector current can be seen. At -75 Oe, the FM2 layer magnetization underneath the tip flips within a few Oersted due to domain wall motion processes and the spin valve is in an antiparallel configuration. In Fig. 6(a), this configuration is visible at point 3. When the external magnetic field is reversed to values below 
-99 Oe, a steep step [seen in Fig. 6(a)] arises and the collector current reaches values corresponding to an almost $90^{\circ}$ magnetization alignment. The local magnetization in the FM2 layer switches via domain wall motion to the $[-110]$ axis, while the FM1 layer is still almost orientated along the [110] axis [Fig. 6(a), point 4]. In this field range, coherent magnetization rotation processes force again the FM1 layer magnetization slowly toward the $[-110]$ axis. Reversing the sign of the sweep, a similar magnetic behavior is obtained. This implies that the magnetization reversal along this intermediate axis is dominated by domain wall motion and simultaneous spin rotation processes. The hysteresis loop shown in Fig. 5(a) is mostly a result of magnetization realignment in the FM2 layer, while the magnetization orientation of the FM1 layer remains always close to the [110] axis. The magnetic field sweep along this intermediate axis inverts more easily the magnetization of the FM2 layer compared to the sweep along the magnetic easy or hard axis.

In conclusion, we have studied the local magnetic behavior at room temperature by means of BEMM. We observe a distinct hot-electron transport behavior by varying an inplane external magnetic field along different in-plane angles with respect to the epitaxial $\mathrm{Fe}_{34} \mathrm{Co}_{66} / \mathrm{Au} / \mathrm{Fe}_{34} \mathrm{Co}_{66}$ spin valve. The magnetic anisotropies and the energy landscape for the magnetizations of the ferromagnetic layers involved were determined by FMR. Both $\mathrm{Fe}_{34} \mathrm{Co}_{66}$ layers possess an energy landscape composed of a four- and a twofold compo- nent. The magnetic easy directions are aligned along the [110] directions for the two ferromagnetic layers. The $\langle 100\rangle$ axes represent magnetic hard axes and the [1-10] directions an intermediate axis, respectively. The determined magnetic anisotropy constants allowed us to simulate the local hysteresis loops obtained by BEMM and the macroscopic hysteresis loops obtained by MOKE as well. In doing so, we were able to calculate the relative magnetization configuration of the spin valve as a function of the external magnetic field and to correlate this reasonably well to the experimental hysteresis loops. Since different magnetization configurations of the spin valve are available, the epitaxial spin valves allow multimagnetocurrent values in different field directions. In addition, we found in the experiments with field sweeps along the easy [110] axis coercive field distributions which are composed of two Gaussian curves for each $\mathrm{Fe}_{34} \mathrm{Co}_{66}$ layer. They indicate the existence of weakly pinned and more strongly pinned types of domains. Further, we found that the magnetization of the $\mathrm{Fe}_{34} \mathrm{Co}_{66}$ layer grown directly onto the semiconductor is inverted at lower magnetic fields when the magnetization reversal is carried out along the easy and hard axes in contrast to reversals along the intermediate axis.

Financial support by the DFG through the SFB 689 is gratefully acknowledged. We thank T. Martin for the possibility to use his simulation program.
${ }^{1}$ G. Binasch, P. Grunberg, F. Saurenbach, and W. Zinn, Phys. Rev. B 39, 4828 (1989).

${ }^{2}$ M. N. Baibich, J. M. Broto, A. Fert, F. Nguyen Van Dau, F. Petroff, P. Etienne, G. Creuzet, A. Friederich, and J. Chazelas, Phys. Rev. Lett. 61, 2472 (1988).

${ }^{3}$ M. Julliere, Phys. Lett. 54A, 225 (1975).

${ }^{4}$ D. J. Monsma, J. C. Lodder, Th. J. A. Popma, and B. Dieny, Phys. Rev. Lett. 74, 5260 (1995).

${ }^{5}$ K. Mizushima, T. Kinno, T. Yamauchi, and K. Tanaka, IEEE Trans. Magn. 33, 3500 (1997).

${ }^{6}$ W. H. Rippard and R. A. Buhrman, J. Appl. Phys. 87, 6490 (2000).

${ }^{7}$ W. H. Rippard and R. A. Buhrman, Appl. Phys. Lett. 75, 1001 (1999).

${ }^{8}$ W. H. Rippard and R. A. Buhrman, Phys. Rev. Lett. 84, 971 (2000).

${ }^{9}$ R. Heer, J. Smoliner, J. Bornemeier, and H. Brueckl, Appl. Phys. Lett. 85, 4388 (2004).

${ }^{10}$ E. Heindl, J. Vancea, and C. H. Back, Phys. Rev. B 75, 073307 (2007).

${ }^{11}$ E. Haq, T. Banerjee, M. H. Siekman, J. C. Lodder, and R. Jansen, Appl. Phys. Lett. 86, 082502 (2005).

${ }^{12}$ R. Vlutters, O. M. J. van 't Erve, S. D. Kim, R. Jansen, and J. C. Lodder, Phys. Rev. Lett. 88, 027202 (2001).

${ }^{13}$ L. D. Bell, W. J. Kaiser, and M. H. Hecht, J. Vac. Sci. Technol. B 9, 594 (1991).
${ }^{14}$ B. G. Park, T. Banerjee, J. C. Lodder, and R. Jansen, Phys. Rev. Lett. 97, 137205 (2006).

${ }^{15}$ V. P. Zhukov, E. V. Chulkov, and P. M. Echenique, Phys. Rev. B 73, 125105 (2006).

${ }^{16}$ S. van Dijken, X. Jiang, and S. S. P. Parkin, Phys. Rev. B 66, 094417 (2002).

${ }^{17}$ T. Banerjee, E. Haq, M. H. Siekman, J. C. Lodder, and R. Jansen, Phys. Rev. Lett. 94, 027204 (2005).

${ }^{18}$ R. Jansen, J. Phys. D 36, R289 (2003).

${ }^{19}$ S. van Dijken, X. Jiang, and S. S. P. Parkin, Appl. Phys. Lett. 83, 951 (2003).

${ }^{20}$ S. van Dijken, X. Jiang, and S. S. P. Parkin, Appl. Phys. Lett. 82, 775 (2003).

${ }^{21}$ C. J. Gutierrez, G. A. Prinz, J. J. Krebs, M. E. Filipkowski, V. G. Harris, and W. T. Elam, J. Magn. Magn. Mater. 126, 232 (1993).

${ }^{22}$ M. Dumm, M. Zoelfl, R. Moosbuehler, M. Brockmann, T. Schmidt, and G. Bayreuther, J. Appl. Phys. 87, 5457 (2000).

${ }^{23}$ M. Dumm, B. Uhl, M. Zoelfl, W. Kipferl, and G. Bayreuther, J. Appl. Phys. 91, 8763 (2002).

${ }^{24}$ B. Heinrich and J. F. Cochran, Adv. Phys. 42, 523 (1993).

${ }^{25}$ B. Heinrich, Ultrathin Magnetic Structures I (Springer, Berlin, 1994).

${ }^{26}$ R. Naik, A. Poli, D. McKague, A. Lukaszew, and L. E. Wenger, Phys. Rev. B 51, 3549 (1995).

${ }^{27}$ C. A. F. Vaz and J. A. C. Bland, Phys. Rev. B 61, 3098 (2000).

${ }^{28}$ From attocube systems, Munich, Germany. 